\title{
Estado actual de la investigación en teoría económica del valor a nivel internacional (2019)
}

\author{
Economic Value Theory: \\ Current International State-of-Art (2019)
}

Angie Vanessa Zambrano-Valencia, Sergio Sánchez Martínez, Mariana Villabona Martínez, Cristian Rincón Cruz, Sebastián Sarmiento Carvajal, Valentina Salazar Méndez y Estiven Aguilera"

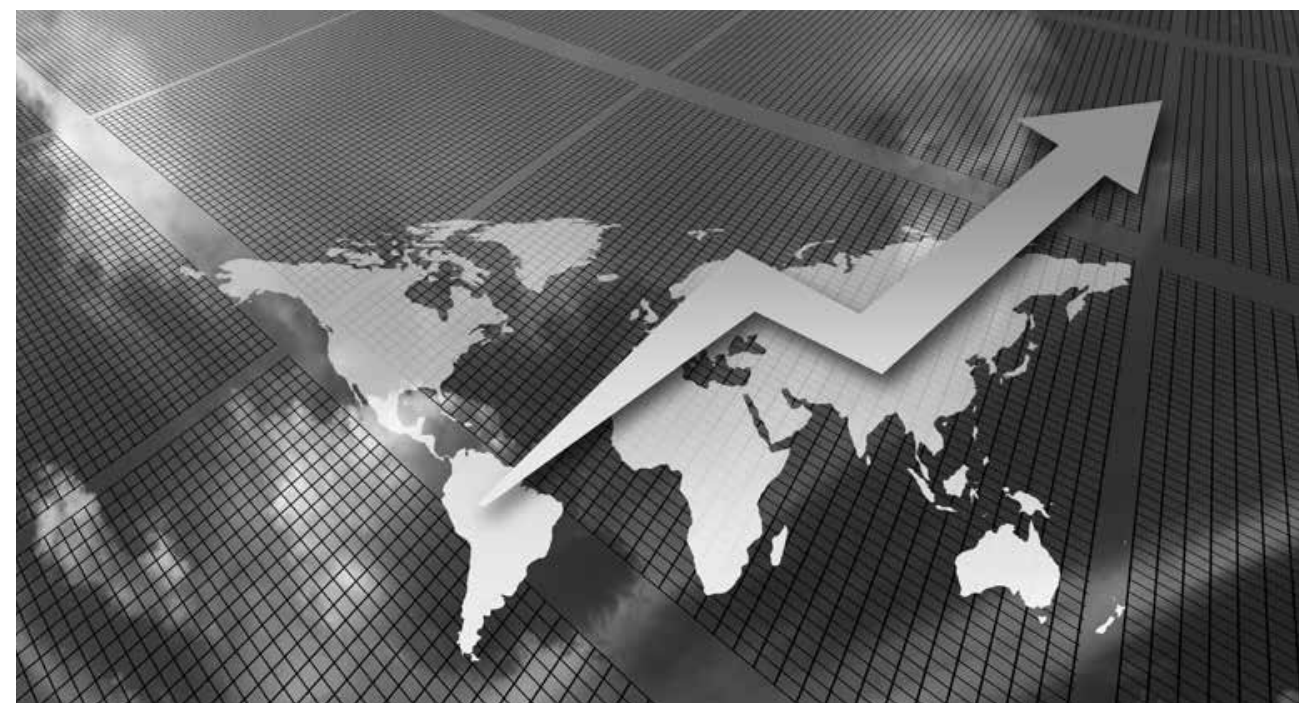

\section{Resumen}

La teoría económica del valor es un área en la que no existe consenso y no ha sido un tema predilecto de investigación en las revistas científicas líderes en ningún periodo de tiempo. Por eso, en este artículo se propone describir el estado actual de

Citar este artículo como: Zambrano, A. V., Sánchez, S., Villabona, M., Rincón, C.,

Sarmiento, S., Salazar, V. y Aguilera, E. (2020). Estado actual de la investigación en teoría económica del valor a nivel internacional (2019). Revista Papeles, 11(22), 72-80.

Fecha de recibido: octubre 10 de 2020 • Fecha de aceptación: noviembre 15 de 2020

* Estudiantes de Economía de la UNAL. Trabajo dirigido por el prof. Enrique Ferrer-Corredor. Correos electrónicos: Angie Vanessa Zambrano Valencia (avzambranov@unal.edu.co); Sergio Alejandro Sánchez Martínez (sesanchezm@unal.edu.co); Mariana Villabona Martínez (mvillabona@unal.edu. co); Cristian Julián Rincón Cruz(crrincon@unal.edu.co); Juan Sebastián Sarmiento Carvajal (jsamientoc@unal.edu.co); Valentina Salazar Méndez (vsalazar@unal.edu.co); Arnold Estiven Aguilera (araguilerar@unal.edu.co). 
investigación en la teoría económica del valor a partir de una evaluación bibliométrica sobre artículos publicados en el 2019 y sus referencias bibliográficas, detectando tendencias en géneros documentales, periodos de tiempo de publicación, concentración geográfica, códigos JEL y palabras clave. Se concluye que la teoría del valor económica tiene una importancia relativamente baja, está escrita predominantemente en lengua inglesa y construida en Europa, con artículos científicos del siglo XXI como fuente predilecta. Con respecto a las referencias, se concentran en Europa y Estados Unidos, evidenciando múltiples corrientes de pensamiento alrededor de esta teoría. Se advierte sobre el papel del continente asiático como uno interesado en esta teoría, que publica en sus lenguas nativas.

Palabras clave: teoría económica, teoría del valor, estudio bibliográfico.

JEL: B00, B41, D46, F01, N01

\begin{abstract}
The value theory is a subject under discussion in economics, and it has not been a salient topic on the leading journals. The present research describes the state-ofart of current research in economic value theory. By bibliometrics, papers of 2019 which aboard this topic, and its references are evaluated, identifying tendences on documentary genre, publishing time-periods, geographical concentrations, JEL codes and Keywords. As final conclusion, the economic theory of value has low importance, is mainly written in English and builded in Europe, with papers of XXI century as favorite source. The references are mainly concentrated in Europe and United States and reflect the multiplicity of schools of thought about economic theory of value. The Asian continent is noted as interested on this field, which publishes on its native languages.
\end{abstract}

Keywords: economic theory, theory of value, bibliographical study.

\title{
Introducción
}

La construcción del conocimiento científico está basado en axiomas o principios compartidos por una comunidad académica, los cuales se ponen a prueba por métodos sistemáticos, contrastando con datos fácticos o argumentos teóricos, y se verifican o falsean (Bunge, 2013). Para la economía como ciencia social, la teoría del valor se constituye como un ejemplo de debate y construcción de la teoría económica (Aspromourgos, 1996; Dobb, 1975; Kurz, 2013), abarcando diferentes concepciones sobre el valor de uso y de cambio de bienes según su utilidad y escasez (Bowley, 1963; Langholm, 2009).
La perspectiva que cada economista adopta sobre esta teoría, explícita o implícitamente, es determinante para un rango amplio de fenómenos económicos, como las fuerzas que determinan los precios, la distribución del ingreso y del excedente, los niveles de empleo y el salario (Aspromourgos, 1996; Bortis, 2003; Bowley, 1963; Dobb, 1975; Hamilton, 1918; Kurz, 2013).

Un ejemplo de esta determinación se observa en la tarea de definir el valor económico de diferentes fragmentos del medio ambiente. Desde un enfoque neoclásico, se realiza a partir de una disponibilidad a pagar, 
de costos y beneficios, dejando de considerar la naturaleza como una externalidad sin costo. Sin embargo, esta forma de atribuir valor económico parece insuficiente y cuestionable. Así, otros enfoques se preguntan por las ventajas y desventajas de establecer un sistema de valoración específico, por la variación de las valoraciones con las fluctuaciones de la economía y por las implicaciones a largo plazo de la mercantilización de la naturaleza. Además, se preguntan por la necesidad de la alineación del valor ecológico con un precio económico, resaltando enfoques que consideran el rol de los sistemas de valores personales que orientan el comportamiento humano hacia la naturaleza (Whitehead, 2014).

Las discusiones sobre la teoría del valor son publicadas principalmente en forma de artículos académicos, los cuales ocupan un rol esencial en la construcción de conocimiento científico por la circulación constante de una cantidad altísima de investigación especializada y calificada (Contreras, Buzeta y Pedraja-Rejas, 2015; Guo et al., 2014). Sin embargo, la teoría del valor no destaca como tema predilecto en las revistas científicas de la economía en ningún periodo de tiempo (Claveau y Gingras, 2016; Karbownik y Knauff, 2009; Kelly y Bruestle, 2011; Kosnik, 2018), lo que resulta curioso, ya que no existe un consenso sobre la validez de la teoría del valor marginalista, corriente neoclásica y más difundida. El debate sigue abierto y es cuestionado desde diferentes puntos de vista. Retomando las palabras de Oscar Wilde en su obra de $E l$ abanico de Lady Windermere, "hoy en día la gente conoce el precio de todo y el valor de nada" (citado en Whitehead, 2014).

\section{Marco teórico alrededor de la teoría del valor}

La literatura científica registra posiciones de autores clásicos y neoclásicos, y autores que retoman sus ideas (Bortis, 2003; Bradby, 1982; Gehrke y Kurz, 2006; Hedoin, 2011; Hicks y Allen, 1934; Karayiannis, 1991; Katz, 2002; y Langholm, 2009). Dentro de los debates presentados destacan diferencias sobre el carácter de la utilidad, por unos definido como objetivo, reconocido en la producción o trabajo del bien o servicio; y por otros como subjetivo, retratado en el grado de satisfacción personal que determina la demanda del bien o servicio (Hedoin, 2011; Karayiannis, 1991; Katz, 2002). Asimismo, existen diferentes concepciones con respecto al desarrollo histórico de las teorías. Los desarrollos de autores clásicos como William Petty, Richard Cantillon, François Quesnay, James Steuart, Adam Smith, David Ricardo y John Stuart Mill, son definidos por la corriente neoclásica ortodoxa como una versión temprana y sin pulir de la teoría marginalista, que luego sería mejorada por autores como William Jevons, Carl Menger, Léon
Walras y Alfred Marshall (Aspromourgos, 1996; Dobb, 1975; Kurz, 2013).

Sin embargo, desde corrientes heterodoxas, se señalan dos características de la disciplina: la economía política clásica, noción acuñada por Karl Marx (Aspromourgos, 1996), y economía "a secas" para los neoclásicos, encerrando dos concepciones distintas, pero complementarias, de construir conocimiento científico (Bortis, 2003). En la primera, el economista teórico se asume como un constructor de modelos puestos a prueba empíricamente, explicando fenómenos económicos a partir de premisas y evidencias. En la segunda, el economista teórico busca destilar los principios y fundamentos que subyacen a las explicaciones, con el ánimo de comprender cómo funcionan esencialmente los sistemas socio-económicos (Bortis, 2003).

Bajo este marco, los economistas políticos clásicos no son asumidos como predecesores de la teoría marginalista, sino como teóricos con principios explicativos propios para la asignación de valor económico y para la 
distribución. Adicionalmente, se añade la importancia de ubicar los principios en un contexto sociohistórico para comprender su transformación (Marx, 2014), pues factores distintos a los intelectuales influyen en el proceso de elección de unas teorías por encima de otras (Mackie, 1998).

En consecuencia, estos enfoques retoman postulados clásicos para debatir axiomas de la teoría neoclásica y demostrar alternativas posibles. Tal es el caso del principio de John Maynard Keynes sobre demanda efectiva (Aspromourgos, 1996; Bortis, 2003), que discutió la ley neoclásica de Jean Baptiste Say y logró un reconocimiento bajo unas condiciones socioeconómicas particulares: la crisis económica de 1929 (Dobb, 1975). Piero Sraffa refleja otro caso ejemplar al retomar el principio de excedente de Ricardo para analizar la sociedad moderna, el valor y la distribución desde una aproximación distinta a la de Marshall, quien también retoma a Ricardo pero desde una corriente neoclásica (Aspromourgos, 1996; Gehrke y Kurz, 2006). Entre los estudiantes de Sraffa destacan Pierangelo Garegnani, Luigi Pasinetti, Krishna Bharadwaj, John Eatwell, Geoff Harcourt, Bertram Schefold e Ian Steedman, quienes mantuvieron un debate sobre la teoría del capital y la distribución con Paul Samuelson, economista clave en el desarrollo de la síntesis neoclásica-keynesiana (Aspromourgos, 1996; Kurz, 2013). Finalmente, un autor clave es Karl Marx y su propuesta de la teoría del valor-trabajo, derivada de la revisión de autores clásicos como Smith y Ricardo (Marx, 2014) ${ }^{1}$.

\section{Producción académica en la economía}

Ahora bien, en lo que respecta al surgimiento de las revistas científicas de economía, estas surgieron un siglo después de la publicación de La riqueza de las naciones (1776) de Adam Smith (Diamond, 1988, citado en Guo et al., 2014). Aunque la aparición de revistas europeas data de 1840 , se resalta con mayor frecuencia el surgimiento de la primera revista profesional de economía inglesa (The Royal Economic Journal, 1891) y la primera estadounidense (Quarterly Journal of Economics, 1886), dado que la mayoría de citaciones en revistas líderes en economía refieren a artículos publicados en inglés (Stigler, Stigler y Friedland, 1995). Con base en esta información, Guo y sus colaboradores (2014) establecen 1890 como la década inicial de la producción académica. Sin embargo, autores clásicos de la teoría del valor ya habían publicado sus escritos en forma de texto, por lo que referencias que datan de 1840 hacia atrás sobre este tema están consignadas en libros y no en artículos científicos.

La economía como disciplina aumenta su producción académica de manera exponencial con el paso de los años, especialmente

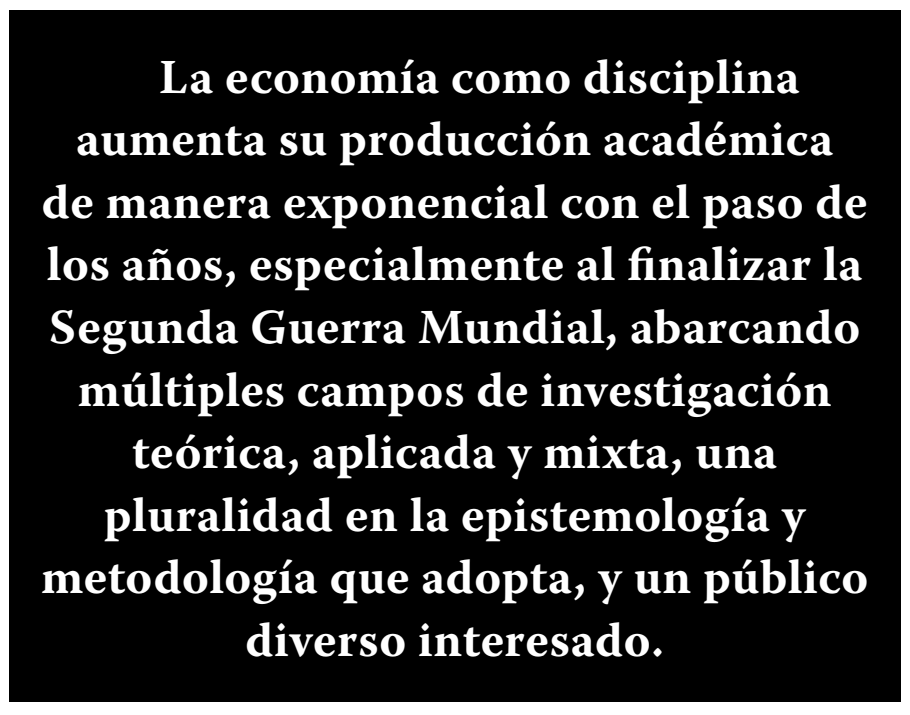

al finalizar la Segunda Guerra Mundial, abarcando múltiples campos de investigación teórica, aplicada y mixta, una pluralidad en la epistemología y metodología que adopta, y

1 Remitirse a Collins (2016) para una revisión reciente del desarrollo de la teoría del valortrabajo. 
un público diverso interesado (Cherrier, 2017; Claveau y Gingras, 2016; Kelly y Bruestle, 2011). Con este panorama, construir un método de clasificación del conocimiento científico económico es un reto, tratando de conciliar demandas internas y externas de la disciplina (Cherrier, 2017). En la actualidad, el sistema de clasificación por códigos del Journal of Economics (JEL Classification es su nombre en inglés) es el resultado de ese proceso y es reconocido por la Asociación Estadounidense de Economía (AEA: American Economic Association). Asimismo, los códigos JEL son ampliamente utilizados tanto por investigadores en diferentes áreas de la economía con consistencia en su uso (Kosnik, 2018), como en investigaciones para explorar el desarrollo de la economía en las revistas científicas (Karbownik y Knauff, 2009; Kelly y Bruestle, 2011), aunque no existe una revisión sobre las publicaciones en el campo de la teoría del valor, cuyo código JEL es el D46.

\section{La bibliometría como método en la economía}

Ante el volumen de información cada vez mayor en la producción académica de la economía (Claveau y Gingras, 2016), los métodos cuantitativos de investigación han adquirido relevancia por su capacidad de procesamiento y análisis (Cherrier y Svorenčík, 2018). Dentro de ellos, la bibliometría permite evaluar calidad e impacto de la literatura científica (Sacho, 1990), al identificar niveles de auto-referenciación (Simoes y Crespo, 2020), coautoría y colaboración internacional (Molina et al., 2016; Orazbayev, 2017; Seltzer y Hamermesh, 2018), geografía y concentración de las autorías (Ek y Henrekson, 2019; Fontana, Montobbio y Racca, 2019), temas centrales y promedio de referencias por año y por artículo (Bornmann y Wohlrabe, 2019).

A partir del marco teórico construido, se encuentra que la teoría del valor no cuenta con principios aceptados por consenso por la comunidad académica económica, centrando su discusión entre autores europeos, a excepción de Krishna Bharadwaj (India) y Paul Samuelson (Estados Unidos). Adicionalmente, se observa que el debate ha surgido en momentos históricos específicos como en la década de 1930, con la gran depresión y la propuesta de Keynes sobre el rol del Estado en la regulación económica. Finalmente, se advierte que la economía es una ciencia publicada principalmente en idioma inglés y la teoría del valor no ha sido uno de los temas mayoritariamente elegidos en las revistas científicas de economía en ningún periodo de tiempo, resultados obtenidos a través de metodologías cuantitativas ampliamente utilizadas (Claveau y Gingras, 2016; Kelly y Bruestle, 2011; Guo et al., 2014).

\section{Objetivo de la investigación}

El objetivo general del presente trabajo es hacer una exploración del estado actual de investigación en la teoría económica del valor, con base en la evaluación bibliométrica de artículos científicos sobre este tema publicados en el 2019. Para esto, se identifica el porcentaje de artículos publicados en revistas científicas indexadas $(<50 \%)^{2}$ y su nivel de importancia relativa en el campo de la economía (bajo); idioma predilecto de publicación (inglés $>50 \%$ ); países a los que pertenecen las revistas y los autores (continente europeo

2 Los valores numéricos y textuales consignados en paréntesis en este párrafo responden a las hipótesis planteadas por los autores de acuerdo a los hallazgos teóricos previamente realizados. 
$>50 \%) ;$ y códigos de clasificación utilizados junto al D46 (B: Schools of Economic Thought and Methodology; D: Microeconomics; E: Macroeconomics and monetary economics; G: Financial economics; J: Labor and demographic economics). Asimismo, se identifica el porcentaje de referencias distribuidas en distintos periodos de tiempo (siglo XX y XXI $>50 \%$ ), porcentaje de artículos en comparación con otros géneros documentales $(>50 \%)$, porcentaje de literatura publicada en inglés ( $>50 \%)$, países y áreas geográficas dominantes (continente europeo y norteamericano $>50 \%$ ) y palabras clave más utilizadas (marginalism, neoclassical economics, labor, economic theory, Smith, Ricardo, Marx, Sraffa, Keynes, Walras, Menger, price). Se puede hipotetizar que la economía es una ciencia de lengua predominantemente inglesa, que la teoría del valor no es registrada por las revistas líderes en economía aunque sus publicaciones se concentran en Europa y Estados Unidos, y que tanto los artículos como sus referencias están asociadas mayormente a conceptos relacionados con precio, finanzas y marginalismo, corrientes dominantes en la actualidad.

\section{Metodología}

La presente investigación tiene un enfoque cuantitativo de alcance descriptivo y con la bibliometría como método. La selección de la muestra se realizó a partir de la búsqueda en Google Scholar con las palabras "JEL CLASSIFICATION" y "D46”, filtrando los resultados para el año 2019, obteniendo un corpus inicial de 29 artículos. Para cada uno de estos artículos, se calculó el número de referencias, obteniendo un corpus final de 1061 (29 artículos y 1032 referencias). Con esta muestra se tuvieron dos etapas:

1. Identificación del nivel de importancia relativa de los artículos sobre teoría del valor en el 2019 a partir de su publicación o no en revista indexada y el cuartil ocupado reportado por $\mathrm{Scimago}^{3}$, concentración geográfica (países y continentes dominantes), idioma predilecto y códigos JEL asociados.

2. Identificación de la vida media de las referencias a partir del promedio de referencias por periodos de tiempo de 100, 10 y 5 años (Colorado y Anaya, 2018), concentración geográfica (países y continentes dominantes), idioma, género documental y palabras clave predilectos; y análisis de

3 Entre más cercano al Q1, más importancia relativa. referencias comunes, indicando que estén bibliográficamente relacionadas $\mathrm{y}$, por ende, su aproximación al campo de conocimiento sea más cercana (Sancho, 1990).

El registro del corpus se realizó en Excel a partir de dos matrices, que posteriormente fueron analizadas a través del software JASP. La matriz de artículos iniciales, con 29 datos, sistematiza información sobre la revista en que fue publicado cada artículo (nombre, idioma, país, continente, indexada o no en scimago, cuartil), sobre el artículo (título, año de publicación, códigos JEL) y sobre los autores (nombres, país de procedencia de acuerdo a nacionalidad o educación superior, afiliación institucional actual, país de residencia actual y continente). Por su parte, la matriz de referencias, con 1032 datos, registra el nombre del artículo inicial, título de la referencia, año de publicación neto y clasificado en periodos de tiempo (siglo, década, quinquenio), género documental (artículo, libro, base de datos, evento académico, tesis, etc.), revista, universidad o editorial en que fue publicada la referencia; idioma, palabras clave, nombre de los autores y país de procedencia (lugar de nacimiento de los autores, de estudios de pregrado o de afiliación institucional actual, de acuerdo a la accesibilidad de la información). 


\section{Resultados}

A partir de la sistematización y procesamiento de datos con el software JASP y NVivo, este último para las palabras clave, se obtuvo un corpus final de 1059 datos (29 artículos iniciales y 1030 referencias). Los dos datos de diferencia con respecto a los planteados en metodología se deben a que dos referencias fueron registradas dos veces en la bibliografía de un mismo artículo. Además, dentro de los 29 artículos iniciales se incluye uno publicado en el año 2017, pues es registrado por Google Scholar como del 2019. Ante esta situación, para futuras investigaciones es necesario revisar los artículos directamente para confirmar su fecha de publicación, pues el motor de búsqueda puede ser inexacto.

A continuación se exponen de forma detallada los resultados arrojados en cada una de las matrices.

\section{Matriz de artículos iniciales}

En general, las hipótesis planteadas respecto a los 29 artículos iniciales sobre teoría del valor se confirmaron. El 48,3\% de los artículos $(14 / 29)$ no se encuentran publicados en revistas clasificadas por Scimago, mientras que 9 de los 15 restantes (31\%) se encuentran distribuidos en los cuartiles 3 y 4 y tan solo 2 artículos (6.9\%) se publicaron en una revista clasificada en el cuartil 1, la revista Cambridge Journal of Economics. La revista Real Estate Management and Valuation, ubicada en el cuartil 3, también contó con 2 de los 29 artículos publicados en el 2019. Con esta información es posible confirmar que el nivel de importancia relativa de la teoría del valor para la economía es baja, pues no es publicada por revistas líderes del campo.

Con respecto a la concentración geográfica de los artículos, se confirma que la mayoría se encuentran publicados en el continente europeo $(19 / 29,65,6 \%)$, cuyos países de proveniencia son Alemania e Inglaterra (con 4 artículos cada uno), Países Bajos, República
Checa y Ucrania (con dos artículos cada uno), y España, Francia, Lituania, Polonia y Rusia (con un artículo cada uno). El segundo continente con mayores publicaciones fue Asia $(27,6 \%)$ con 8 artículos provenientes de Turquía (2/8), India, Indonesia, Irak, Pakistán, Singapur y Taiwán (con un artículo cada uno). Finalmente, América del Norte presentó la más baja participación con tan solo 2 artículos del total (6,9\%) provenientes de Canadá. Adicionalmente, cabe mencionar que los artículos indexados provienen del continente europeo y asiático, y no del norteamericano.

Ahora bien, el idioma predilecto fue el inglés, encontrando que el 72,4\% del total de artículos iniciales están publicados en esta lengua, y que el 100\% de los artículos publicados en revistas indexadas están en este idioma. El continente norteamericano publicó sus dos artículos en este idioma, mientras que el europeo 15 de los 19 y el asiático 4 de los 8 . Los idiomas distintos utilizados en Europa fueron el checo, el español, el ruso y el ucraniano. En el caso de Asia, fueron el indonesio, el árabe y el turco, este último con dos artículos. En consecuencia, se confirma que el inglés es el idioma predominante en la construcción de conocimiento científico alrededor de teoría del valor para el año 2019, aunque el continente asiático tiene una mayor variabilidad de idiomas utilizados.

Una variable adicional analizada fue el número de autores por artículo, con el ánimo de observar si existe una predilección por la autoría individual o la colaborativa. Así, se encuentra que 13 de los 29 artículos (44,8\%)

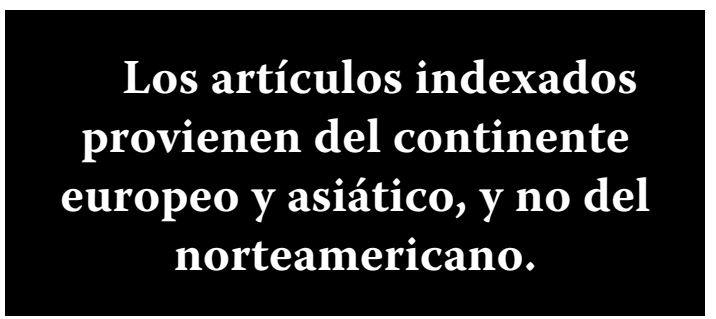


están escritos por un solo autor, mientras que el 55,2\% restante está escrito en coautoría. De aquellos escritos en colaboración se encuentra que 9/29 tienen 3 autores, 5/29 tienen 2 autores y $2 / 29$ tienen cuatro. Sin embargo, al realizar un análisis cruzado con el nivel de importancia relativa del artículo, determinado por el cuartil de Scimago, no se encuentra que exista alguna relación. Caso opuesto al de la variable continente, en el que se encuentra que Europa tiene una mayor predilección por la autoría individual (11/19), en comparación con Asia (2/8) y con Norteamérica (0/2).

Con relación a la concentración geográfica de los autores, si bien se confirma que mayoritariamente están ubicados en el continente europeo (28/58, 48,3\%), Asia tiene una presencia significativa del 41,4\% (24/58). Respecto a los países europeos con mayor concentración de autores, encontramos a Ucrania y República Checa (cuatro autores cada uno), Eslovaquia, Italia y Polonia (con tres autores cada uno) y España e Inglaterra con dos autores cada uno. Los siete autores restantes están ubicados en Alemania, Chipre, Francia, Grecia, Lituania, Rusia y Suiza. En el caso de Asia, Taiwán concentra a cuatro autores, mientras que Turquía, Pakistán, Malasia, Japón, Irak e Indonesia concentran a tres autores cada uno. Los dos autores restantes habitan en Omán e India. Asimismo, se encontró un autor oceánico ubicado en Australia, dos africanos ubicados en Nigeria y tres norteamericanos ubicados en Canadá.

La última categoría analizada en la matriz de artículos iniciales corresponde a los códigos JEL que acompañaban al D46, confirmando la presencia de códigos pertenecientes a la letra D (Microeconomía), con 18 de los 83 códigos totales (21,69\%), y a la B (Historia del pensamiento económico, metodología y aproximaciones heterodoxas) con 11/83 (10,84\%). Un resultado significativo no esperado fue la presencia de 9 códigos (10.84\%) pertenecientes a la $\mathrm{C}$ (Métodos Matemáticos y Cuantitativos), comprobando lo expuesto por autores como Backhouse y Cherrier (2017) y Cherrier \& Svorenčík (2018) sobre un giro cuantitativo y aplicado en la economía. En cuanto a las subcategorías de JEL presentadas con mayor frecuencia fueron la B12 (Clásicos) y B13 (Neoclásicos), permitiendo hallar una relación entre los temas de teoría del valor y las corrientes de pensamiento clásico y neoclásico y sus respectivos teóricos, así como con las metodologías cuantitativas.

\section{Matriz de referencias}

En el caso de las referencias también fue posible confirmar varias hipótesis. En primer lugar, se observa que el género documental predilecto de las referencias fue el artículo científico, contando con 634 de las 1030 (61,6\%). En segundo lugar, los autores consultaron los libros (284/1030: 27,6\%) o capítulos de libros en 24/1030 (2,3\%). Además, se consultaron documentos oficiales (42/1032: 4,1\%), bases de datos (13/1030: $1,3 \%)$ y ponencias de eventos académicos (12/1039: 1,2\%). Es notable observar que con poca frecuencia se consultaron tesis de doctorado, maestría o pregrado, cuya sumatoria solo representa 10/1030, aunque comienzan a ser citadas recién en el siglo XXI.

El periodo de tiempo de las referencias de autores utilizadas en los artículos de teoría

Figura 1. Distribución de la cantidad de referencias de acuerdo al año de publicación

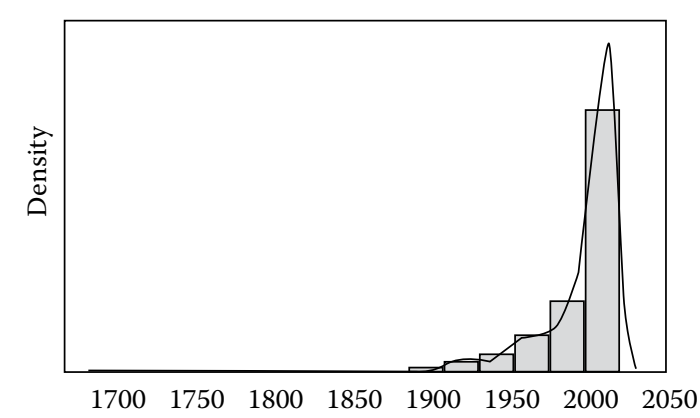

Fuente: elaboración propia. 
del valor del 2019 abarca desde el siglo XVIII hasta el XXI, con un crecimiento exponencial a mayor paso del tiempo, comenzando con $4 / 1030$ referencias en el siglo XVIII y terminando con 680/1030 en el siglo XXI. Las referencias que fueron publicadas en el siglo XVII corresponden a dos obras del autor clásico Adam Smith, una de Anne Robert Jacques Turgot (cofundador de la escuela fisiócrata) y una de Francois Quesnay (fisiócrata y predecesor de la escuela clásica). Las referencias del siglo XIX fueron 10/1030 y corresponden a obras de autores fundadores de la teoría neoclásica como Jean Baptiste Say, Vilfredo Pareto, Hermann Gossen, León Walras, Alfred Marshall y William Stanley Jevons, así como a una obra del autor clásico David Ricardo y dos pertenecientes a la teoría del valor-trabajo: Karl Marx y Johann Karl Rodbertus. Cabe resaltar que estas referencias están consignadas en libros y se concentran en dos de los 29 artículos publicados en el 2019 (Eatwell, 2019; Ibraev, 2019).

Ahora bien, todos los artículos científicos referenciados datan del siglo XX (164: 25,9\%) y XXI (470: 74,1\%), siendo citados con mayor recurrencia aquellos pertenecientes a la década del 2010 (301/634: 47,5\%), y en el quinquenio del 2006 (99/634: 15,6\%). En el caso de los libros, los más citados provienen del siglo XX y XXI, cada periodo con proporciones exactamente iguales (135/284: 47,5\%) y en su mayoría fueron publicados desde el 2001 hasta la actualidad (133/284), y en el quinquenio del 2010 (39/284). Fuera de este periodo, el quinquenio de 1955 y 1961 presentan fracciones representativas, con $12 / 284(4,2 \%)$ y $14 / 284$ $(4,9 \%)$ respectivamente.

Respecto a las revistas, editoriales o instituciones que publicaron mayoritariamente las referencias, destacan la American Economic Review (14/1030: 1,4\%), Cambridge University Press (12/1030: 1,3\%), Econométrica (13/1030: 1,3\%), Insurance: Mathematics and Economics y Oxford University Press (con 12/1030: 1,2\% cada una), The Journal of Finance (11/1030:
1,1\%) y Routledge (10/1030: 0,98\%), siendo todas ellas reconocidas en el campo de la economía a nivel mundial.

Con relación al idioma utilizado en las referencias, se confirma la hipótesis de predominancia del inglés, rebasando a los demás con una proporción de 858/1030 (83,30\%). El segundo idioma más utilizado fue el turco con $48 / 1030(4.66 \%)$ y el tercero el ruso con $35 / 1030$ (3.40\%), proporciones notablemente menores en comparación con el inglés. Del mismo modo que en el caso de los artículos de teoría del valor del 2019, se confirma que el idioma predilecto de las fuentes de información consultadas para investigar sobre este tema en la actualidad es el inglés, evidencia a favor de la afirmación de la ciencia económica como una ciencia escrita en esta lengua.

Las palabras clave utilizadas dentro de las referencias, cuya importancia radica en la accesibilidad a trabajos relacionados con un campo específico de investigación en el marco de búsquedas bibliográficas en grandes cantidades de datos (Granda, García y Callol, 2003), confirmó parcialmente la hipótesis. Por un lado, las palabras más comunes estuvieron relacionadas con la teoría económica neoclásica, involucrando descriptores relacionados con capital financiero, mercados, precios, riesgo, inversión, liquidez, consumidor, entre otras. En la figura 2 se visualizan aquellas más frecuentes, así como el idioma y el género documental predilecto. Por otro lado, sin embargo, se rebatió la hipótesis respecto a encontrar descriptores asociados a autores clásicos y neoclásicos o a escuelas de pensamiento económico, hallando en cambio palabras asociadas a la construcción de modelos económicos y medidas del comportamiento del mercado, en línea con lo encontrado en la matriz de artículos iniciales y los códigos de la letra C (Métodos matemáticos y cuantitativos).

Sobre los autores de las referencias se evidencian varios aspectos. Por un lado, se observa una diferencia de 120 artículos entre las 
Figura 2. Palabras clave, género documental, idioma y quinquenios predilectos en las referencias utilizadas para la investigación sobre teoría del valor en el 2019

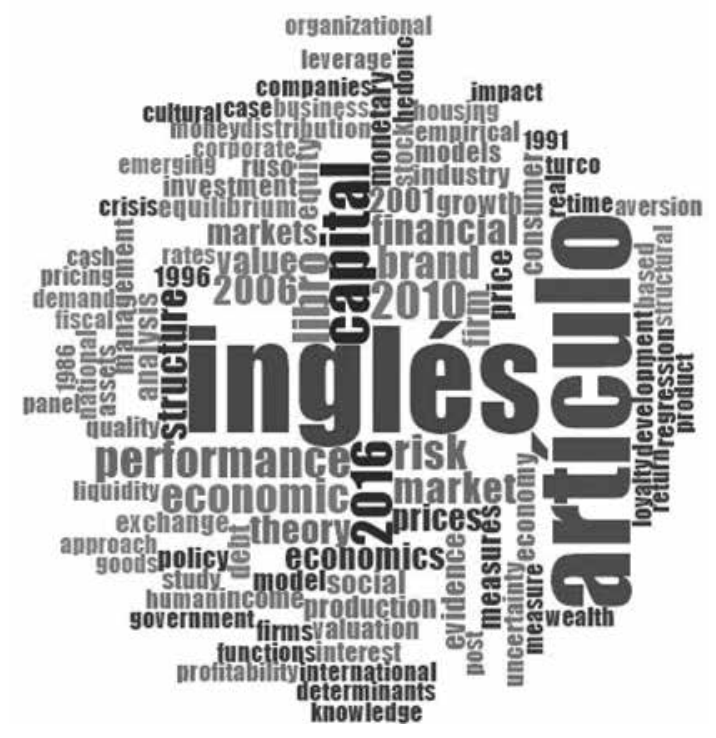

Fuente: elaboración propia.

referencias con autorías individuales (575/1030: $55,9 \%)$ y con coautorías (475/1030: 44,1\%), pareciendo predominar la autoría individual sobre la colaborativa. Por otro lado, se confirma la mayor concentración geográfica de autores referenciados en Europa (736/1755: 41,9\%), distribuyéndose principalmente en Inglaterra (148/736: 20,1\%), Francia (85/736: 11,5\%), Alemania (75/736: 10,2\%), España (55/736: 7,5\%), Italia (51/736: 6,9\%), Polonia (45/736: 6,1\%), República Checa (39/736: 5,3\%), Grecia (27/736: 3,7\%), Suiza (25/736) y Suecia y Países Bajos (20/736 cada uno; $2,7 \%$ cada uno).

El segundo continente con mayor concentración geográfica fue América del Norte (551/1755: 31,4\%), resaltando que el $94,2 \%$ de estos autores están ubicados en Estados Unidos (519/551), representando 3,5 veces la cantidad de autores ubicados en Inglaterra, segundo país a nivel mundial con mayor concentración. Esta información confirma también la hipótesis de mayor concentración geográfica de autores referenciadas en el continente norteamericano.

El continente asiático, por su parte, concentra el 19,8\% (347/1755) de los autores referenciados, alcanzando a ser aproximadamente la mitad de los autores europeos, representando un porcentaje significativo no esperado. Los autores referenciados asiáticos están ubicados principalmente en China (62/347: 17,9\%), Pakistán (42/347: 12,1\%), Turquía (35/347: 10,1\%), Rusia (30/347: 8,6\%), Malasia (29/347: 8,4\%), India (23/347: 6,6\%) y Corea del Sur (19/347: 5,5\%).

Los porcentajes más bajos de concentración están en América Latina ( $0,8 \%)$, en donde Chile concentra el 50\% de los autores (7/14); África (2,8\%), en donde Nigeria concentra el $48 \%$ (24/50) y Kenia el 20\% (10/50); y Oceanía (3,2\%), en donde Australia concentra a 50 de 55 autores oceánicos (90,1\%). En la Figura 3 se puede observar la distribución de autores alrededor del mundo de acuerdo con el grado de concentración geográfica, indicado por el gradiente del color.

Respecto a las referencias compartidas entre distintos artículos publicados en el 2019, se encontraron seis referencias. La obra más citada fue El capital de Karl Marx, en 4 artículos diferentes, evidenciando la relevancia de la teoría del valor-trabajo dentro de la investigación sobre teoría del valor. Producción de mercancías por medio de mercancías de Piero Sraffa le siguió, siendo citada por tres artículos distintos, y es una obra caracterizada por su crítica al modelo neoclásico de los rendimientos a escala, retomando aspectos de David Ricardo y constituyendo lo que hoy se denomina la escuela neoricardiana. Del mismo modo, con tres artículos que la utilizan como referencia, encontramos la obra Value and Capital de John Hicks, considerada exponente de la síntesis neoclásica-keynesiana, en la cual se plantea el compendio de las ideas de Keynes en modelos como IS-LM (o modelo de Hicks-Hansen) y retoma la teoría del valor subjetivo de la escuela neoclásica de la mitad 
Figura 3. Procedencia de autores referenciados en investigación sobre teoría del valor en el

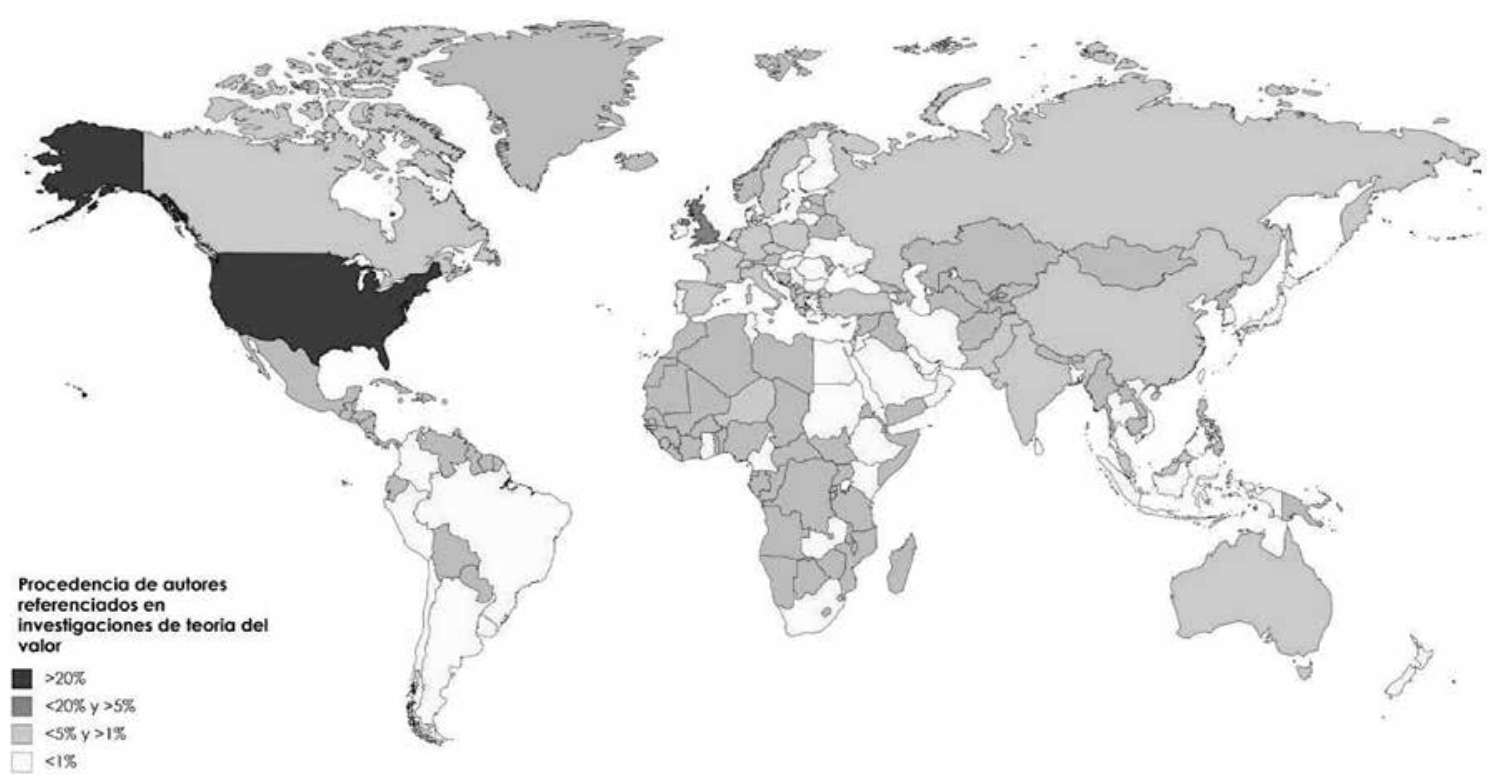

Fuente: elaboración propia.

del siglo XIX. La última referencia común, citada por dos artículos, fue The Empire of Value: A New Foundation for Economics de André Orléan, cuyo aporte es una aproximación a la teoría del valor desde la actualidad, tomando el desarrollo tecnológico y la globalización desde el concepto de valor para explicar las crisis de la economía mundial.

Cabe mencionar que los libros Stocks, Bonds, Bills E Inflation, (de Roger G. Ibbotson y Rex Sinquefield) y Valuing a Business: The Analysis and Appraisal of Closely Held Companies (de Shannon P. Pratt, Robert F. Reilly \& Robert P. Schweihs) también fueron citados dos veces pero esta vez por un mismo artículo perteneciente a India.

Los autores más citados en los artículos de teoría del valor y con un máximo de 8 referencias diferentes fueron: José Antonio Sacristán, médico español que ha contribuido al estudio del valor de los medicamentos y las innovaciones médicas, y Theodore Mariolis, profesor de economía de la universidad de Panteion en Grecia, quien destaca por su aproximación al tema de la distribución, los salarios y la economía política. Con respecto a ambos autores, las 8 referencias están incluidas dentro de un mismo artículo publicado en el 2019 de su propia autoría, representando el $13,4 \%$ de las referencias utilizadas $(8 / 58)$ en el caso de Sacristán (Sacristán y Dilla, 2019), y el $18,6 \%(8 / 43)$ en el caso de Mariolis (2019). De acuerdo a Simoes y Crespo (2020), esto puede representar el liderazgo científico del autor en un campo específico, indicando una investigación continua de un autor o un equipo de trabajo que involucra retomar investigaciones propias pasadas y presentes.

Con una cantidad de 7 referencias, resalta Bruno Frey, economista suizo que ha contribuido académicamente al estudio de la economía del comportamiento y de los incentivos, referencias también citadas dentro de un artículo del 2019 de su propia autoría (Frey, 2019) y que representan el 35\% (7/20) del total de referencias utilizadas.

Con 6 referencias diferentes, destaca Jens Beckert, reconocido sociólogo alemán interesado en el carácter social de la economía y en el estudio de la regularización de los mercados. En este caso, 4 de las 6 referencias están concentradas en un artículo de su misma 
autoría (Beckert, 2019) y representan el 6,2\% del total de referencias de dicho artículo (4/65), correspondiente a uno de los publicados en el Cambridge Journal of Economics. John Hicks, destacado economista inglés que expuso la síntesis del pensamiento neoclásico con las ideas de Keynes a la ciencia económica, también tuvo 6 referencias distintas, de las cuales 3 de ellas fueron citadas en un mismo artículo dedicado a evaluar su punto de vista sobre las expectativas de precio en modelos de equilibrio neowalrasianos (Fratini, Levrero \& Ravagnani, 2019). Con la misma cantidad de 6 referencias se encontró a Piero Sraffa, de origen italiano y de aproximación heterodoxa neoricardiana, quien contribuyó a la crítica de la visión marginalista y a la teoría del valor subjetivo desde el estudio de la producción de mercancías. De estas 6 referencias, 3 se encuentran en un artículo de John Eatwell (2019), uno de los estudiantes de Sraffa más reconocidos.

Con 5 referencias, se encuentra a autores como Frederic S. Mishkin, reconocido economista estadounidense enfocado en política monetaria y mercado financiero y asesor de entidades como el FMI y el BID, representando el 14,3\% (5/35) de un artículo europeo sobre esta temática (Žukauskas y Hülsmann, 2019). Asimismo, Gerard Debreu, economista nacido en Francia pero nacionalizado estadounidense, interesado en la teoría del equilibrio general de Walras. Cabe decir que 4 de las 5 referencias a Debreu están consignadas en el artículo de John Eatwell (2019), un autor heterodoxo, evidenciando una discusión entre dos perspectivas contrarias. Karl Marx, reconocido por la teoría del valortrabajo y su crítica a la propiedad capitalista de los medios de producción, también contó con 5 referencias, todas ellas consignadas en artículos diferentes. Lefteris Tsoulfidis es un autor con 5 referencias, compartidas con Theodore Mariolis, siendo coautor de sus trabajos. La Federación Polaca de Sociedades de Valuadores de la Propiedad también contó con 5 referencias, siendo el único actor institucional destacado dentro de autores más citados aunque dentro de un mismo artículo (Gawron, Yakymchuk y Tyvonchuk, 2019). Pierangelo Garegnani, economista italiano de la escuela neoricardiana y estudiante de Sraffa, entra dentro de los autores con 5 referencias, 4 de ellas ubicadas en el artículo de Eatwell (2019). Finalmente, se encontró a Roy Radner, reconocido por su aporte en la extensión del modelo de Arrow-Debreu hacia el equilibrio de Radner en el contexto del equilibrio general. Las cinco referencias a Radner se encuentran en el artículo de Fratini, Levrero y Ravagnani (2019) sobre modelos de equilibrio neowalrasianos.

Dentro de otros autores citados en menor medida, con 4 referencias, cabe destacar a los siguientes. John Maynard Keynes, influyente economista británico que destaca por sus aportes al sistema económico mundial como la importancia de la intervención estatal en la economía y el estímulo de la demanda por efectos multiplicadores en los agregados macroeconómicos, tanto así que "su argumento de que los gobiernos pueden usar políticas estabilizadoras para prevenir o contrarrestar las declinaciones económicas fueron bautizadas colectivamente como la revolución keynesiana" (Larraín y Sachs, 2002, p. 16). Friedrich von Hayek, reconocido exponente de la escuela austriaca de economía y destacado detractor de las ideas de Keynes por su discrepancia sobre el rol del estado en la economía, defendiendo la idea de la autorregulación del mercado como mecanismo de asignación de precios y criticando el sistema de economía planificada por ser ineficiente y totalitario. Y Ben Bernanke, economista estadounidense que se desempeñó como director de la reserva federal durante la gran recesión de 2008, reconocido por efectuar drásticas políticas monetarias durante la crisis, retomando ideas de Keynes para estimular la demanda agregada a través del consumo. 


\section{Discusión}

Debido a que el presente estudio es de carácter exploratorio, de alcance descriptivo y sobre un periodo de tiempo definido, sus resultados no pretenden generalizar sobre toda la producción académica de la ciencia económica. Sin embargo, los resultados permiten señalar varios aspectos.

En primer lugar, aunque varios artículos se referían a aplicaciones sobre teoría del valor, dentro de las referencias utilizadas se encuentran autores representantes de diferentes escuelas de pensamiento económico alrededor de la teoría del valor, evidenciando la ausencia de un consenso en este campo de la ciencia económica. Así, encontramos autores como Alfred Marshall, Vilfredo Pareto, William Jevons, Carl Menger y Léon Walras (neoclásicos); a Carl Menger y Friedrich Hayek (escuela austriaca); John Maynard Keynes, Joseph Stiglitz y Ben Bernanke (nueva escuela keynesiana); Karl Marx (teoría del valor-trabajo); Theodore Mariolis, Pierangelo Garegnani y Piero Sraffa (neoricardianos); y Thorstein Veblen (institucionalista).

En segundo lugar, las publicaciones con mayor importancia relativa realizadas en el 2019 se enmarcan en una posición alternativa a la teoría marginalista. Por un lado, Cambridge Journal of Economics es una revista de tradición heterodoxa, clasificada por Scimago en el cuartil Q1 y fundamentada en las tradiciones

\section{Se observa que las referencias}

utilizadas en los artículos de

economía referentes a la teoría del

valor son en su mayoría de la década

del 2010, en el caso de los artículos;

y de la década del 2000 para el caso

\section{de los libros.}

de Marx, Keynes, Kalecki, Joan Robinson y Kaldor (Oxford University Press Website, s.f). Por otro lado, Real Estate Management and Valuation, de cuartil Q3, es una revista alemana que busca nuevos conocimientos teóricos y prácticos para mejorar la comprensión en el campo de la valoración, el análisis y la gestión de propiedades inmobiliarias (Sciendo Website Remav, s.f) .

En tercer lugar, se confirman los hallazgos de la literatura sobre el incremento exponencial de los artículos científicos en el siglo XX, siendo estos elegidos mayormente como fuente de consulta sobre el estado actual del conocimiento científico en la economía. En esta misma línea, se confirma la prevalencia del inglés, pues fue el lenguaje predilecto tanto para los artículos como para sus referencias, y fue el idioma de los artículos indexados por la revista Scimago. Así, siguiendo a Stigler et al. (1995), la economía sigue siendo una ciencia de lengua inglesa.

En cuarto lugar, se confirma la concentración de artículos iniciales en el continente europeo, aunque en el caso norteamericano fue contrario a lo esperado, pues presentaron una baja participación. Esto puede deberse a la valoración de la relevancia que pueda otorgarse a la teoría del valor, en el marco de asumir que la teoría marginalista es dominante y ampliamente aceptada, mientras que los autores clásicos son evaluados como predecesores ya superados (Aspromourgos, 1996; Dobb, 1975; Kurz, 2013).

En quinto lugar, se destaca la presencia sorprendente de Asia como continente significativo en la publicación de artículos sobre teoría del valor y en los autores referenciados, evidenciando un interés de esta región en discusiones epistemológicas sobre teoría del valor y sus implicaciones prácticas, un asunto no revelado en la revisión teórica inicial. Sobre esto cabe decir que el acceso a bibliografía de este continente es difícil para el hemisferio de 
occidente, pues sus contenidos están en sus lenguas nativas que no son dominantes en esta área geográfica y las normas de citación que manejan no son las utilizadas tradicionalmente en la academia (por ejemplo, normas APA). Así, supone también un reto tanto para occidente como para oriente el desarrollar un sistema de comunicación que permita la construcción del conocimiento científico superando la barrera del idioma.

En sexto lugar, se observa que las referencias utilizadas en los artículos de economía referentes a la teoría del valor son en su mayoría de la década del 2010, en el caso de los artículos; y de la década del 2000 para el caso de los libros. Ante esta información, es posible enunciar que la vida media de la bibliografía sobre teoría del valor está estipulada en el siglo XXI, volviendo obsoletas aquellas publicaciones realizadas previamente. Esto puede explicarse por fenómenos como la difusión del internet, que eliminó barreras de acceso al conocimiento y aumentó las oportunidades de publicación, contenidos que mayoritariamente pertenecen al siglo actual. Sin embargo, en el contexto de la teoría del valor resulta preocupante por clasificar de obsoleto incluso bibliografía de autores de la economía política clásica, afirmación que debe ser explorada en futuras investigaciones.

\section{Conclusiones y recomendaciones}

A partir de toda la investigación realizada, es posible concluir que la economía es una ciencia escrita predominantemente en lengua inglesa, con base fundamental de referencia a los artículos científicos. Adicionalmente, se concluye que la importancia relativa de la teoría del valor para el campo de la economía es baja, ya que la mayoría de artículos identificados pertenecen a revistas no indexadas $y$ clasificadas en cuartiles bajos, confirmando que no es tema ampliamente publicado en revistas líderes. Finalmente, se concluye que la teoría del valor es un campo construido en el continente europeo principalmente, aunque Asia también demuestra un amplio repertorio de investigación, con el idioma como barrera de acceso. Finalmente, las referencias se encuentran concentradas en Europa y Estados Unidos y están asociadas a conceptos tales como capital, riesgo, mercado, marca, consumidor, demanda, asignación de precios, inversión, entre otras, demostrando que las referencias utilizadas abordan temáticas dentro de un marco predominantemente marginalista neoclásico ortodoxo.

Se sugiere explorar en estudios futuros la relevancia que realmente tienen los autores clásicos como consecuencia de asumir que son obsoletos y que no predominan en la investigación reciente. Asimismo, se sugiere realizar una investigación a profundidad de hechos relevantes dentro de la historia económica mundial (crisis económicas, recesiones, la gran depresión) y los picos de publicaciones científicas, arrojando evidencia sobre si la teoría del valor es relevante en dichos contextos (Dobb, 1975).

Adicionalmente, se sugiere analizar los índices de aislamiento, contrastando las diferencias significativas entre la procedencia y la afiliación actual de los autores acerca de la teoría del valor. Esto debido a que en la construcción de las matrices se encontró que varios de los autores más citados son citados por sí mismos. Además, aquellos artículos escritos en coautoría muestran relaciones tutor académico-estudiante, compañeros de estudio durante el pregrado o posgrado, etc. A este respecto, se sugiere avanzar en la metodología de análisis de redes sociales que puede ser útil para dar cuenta de dichos vínculos entre los investigadores que están construyendo el conocimiento sobre teoría del valor (Cherrier y Svorenčík, 2018). 


\section{Referencias}

Aspromourgos, T. (1996). On the origins of classical economics: distribution and value from William Petty to Adam Smith. Routledge.

American Economic Association. (2019). JEL Classification System / EconLit Subject Descriptors.

Backhouse, R. E., y Cherrier, B. (2017). The age of the applied economist: the transformation of economics since the 1970s. History of Political Economy, 49(Supplement), 1-33.

Beckert, J. (2019). Markets from meaning: quality uncertainty and the intersubjective construction of value. Cambridge Journal of Economics, (published online August 16).

Bornmann, L., y Wohlrabe, K. (2019). Normalisation of citation impact in economics. Scientometrics, 120(2), 841-884.

Bowley, M. (1963). Some seventeenth century contributions to the theory of value. Economica, 122-139.

Bradby, B. (1982). The remystification of value. Capital \& Class, 6(2), 114-133 Bunge, M. (2013). La ciencia: su método y su filosofía. Pamplona: Laetoli.

Cherrier, B. (2017). Classifying economics: A history of the JEL codes. Journal of economic literature, 55(2), 545-79.

Cherrier, B., y Svorenčík, A. (2018). The quantitative turn in the history of economics: promises, perils and challenges. Journal of Economic Methodology, 25(4), 367-377.

Claveau, F., y Gingras, Y. (2016). Macrodynamics of economics: A bibliometric history. History of Political Economy, 48(4), 551-592.

Collins, J. L. (2016). Expanding the labor theory of value. Dialectical Anthropology, 40(2), 103-123.

Colorado, Y. S., y Anaya, O. P. (2018). La evaluación de la actividad científica: Indicadores bibliométricos. In Cienciometría y bibliometría. El estudio de la producción científica: Métodos, enfoques y aplicaciones en el estudio de las Ciencias Sociales (pp. 96-118). Corporación Universitaria Reformada.

Contreras, F. G., Buzeta, L. P., y Pedraja-Rejas, L. (2015). Importancia de las publicaciones académicas: algunos problemas y recomendaciones a tener en cuenta. Idesia (Arica), 33(4), 111-119.

Dobb, M. (1975). Teoría del valor y de la distribución desde Adam Smith; versión en español de Siglo XXI Argentina Editores. SA, Buenos Aires.

Eatwell, J. (2019). Cost of production' and the theory of the rate of profit. Contributions to Political Economy, 38(1), 1-11.

Ek, S., y Henrekson, M. (2019). The Geography and Concentration of Authorship in the Top Five: Implications For European Economics. Scottish Journal of Political Economy, 66(2), 215-245.

Escorcia Otálora, T. A. (2008). El análisis bibliométrico como herramienta para el seguimiento de publicaciones científicas, tesis y trabajos de grado (Doctoral dissertation, Pontificia Universidad Javeriana).

Fratini, S. M., Levrero, E. S., y Ravagnani, F. (2019). Price expectations in neo-Walrasian equilibrium models: Assessing the developments of Hicks's standpoint. Cahiers d'économie Politique/Papers in Political Economy, (1), 117-149.

Frey, B. S. (2019). Awards in the digital world. International Review of Economics, 66(1), 29-36.

Fontana, M., Montobbio, F., y Racca, P. (2019). Topics and Geographical Diffusion of Knowledge in Top Economic Journals. Economic Inquiry, 57(4), 1771-1797.

Gawron, K., Yakymchuk, A., y Tyvonchuk, O. (2019). The bankrupt entity's assets valuation methods: Polish approach. Investment Management \& Financial Innovations, 16(3), 319. 
Gehrke, C., y Kurz, H. D. (2006). Sraffa on von Bortkiewicz: reconstructing the classical theory of value and distribution. History of Political Economy, 38(1), 91-149.

Granda Orive, J. I. D., García Río, F., y Callol Sánchez, L. (2003). Importancia de las palabras clave en las búsquedas bibliográficas. Revista española de salud pública, 77, 765-767.

González de Dios, J., Moya, M., y Mateos Hernández, M. A. (1997). Indicadores bibliométricos: Características y limitaciones en el análisis de la actividad científica Justificación de la evaluación científica. An Esp Pediatr, 47, 235-244.

Guo, S., Zhang, G., Ju, Q., Chen, Y., Chen, Q., y Li, L. (2015). The evolution of conceptual diversity in economics titles from 1890 to 2012. Scientometrics, 102(3), 2073-2088.

Hamilton, W. H. (1918). The place of value theory in economics: I. Journal of Political Economy, 26(3), 217-245.

Hedoin, C. (2011). The Economics of Carl Menger.

Hicks, J. R., y Allen, R. G. (1934). A reconsideration of the theory of value. Part I. Economica, 1(1), 52-76.

Ibraev, L. (2019). The Necesite Quantum Theory of Prices and Money. Disponible en: http://leonard-i-braev.ru/14nec.pdf

Karayiannis, A. D. (1991). Sir James Steuart on Value and Prices. Panepistemio< Peiraieus $>$ : Spudai, 41(2), 167-190.

Karbownik, K., y Knauff, M. (2009). On Importance Of Main Economic Categories: JEL Codes Analysis. Ekonomia: Rynek, Gospodarka, Społeczeństwo, 24, 153-174.

Katz, C. (2002). La actualidad de la teoría objetiva del valor. Filosofía, política y economía en el Laberinto, (9), 89-104.

Kelly, M. A., y Bruestle, S. (2011). Trend of subjects published in economics journals 1969-2007. Economic Inquiry, 49(3), 658-673.

Kosnik, L. R. (2018). A survey of JEL codes: What do they mean and are they used consistently?. Journal of economic surveys, 32(1), 249-272.

Kurz, H. D. (2013). The Theory of Value and Distribution in Economics. Discussions between Pierangelo Garegnani and Paul Samuelson, London and New York: Routledge.

Langholm, O. (2009). Olivi to Hutcheson: tracing an early tradition in value theory. Journal of the history of economic thought, 31(2), 131-141.

Larraín, F., y Sachs, J. D. (2002). Macroeconomía en la economía global. Buenos Aires, Argentina: Pearson Educación.

Mackie, C. D. (1998). Canonizing Economic Theory: How Theories and Ideas are Selected in Economics: How Theories and Ideas are Selected in Economics. Routledge.

Mariolis, T. (2019). The location of the value theories in the complex plane and the degree of regularity-controllability of actual economies. Munich Personal RePEc Archive. Disponible en: https://mpra. ub.uni-muenchen.de/96972/1/MPRA_ paper_96972.pdf

Marx, K. (2014). El capital, crítica de la economía política. México,Fondo de Cultura económica.

Molina, J. A., Alcolea, A., Ferrer, A., Iñiguez, D., Rivero, A., Ruiz, G., y Tarancón, A. (2016). Co-authorship and academic productivity in economics: Interaction maps from the complex networks approach.

Orazbayev, S. (2017). Diversity and collaboration in Economics.

Oxford University Press (s.f.) Cambridge Journal of Economics. Oxford University Press Website.

Sancho, Rosa. (1990) Indicadores bibliométricos utilizados en la evaluación de la ciencia y la tecnología. Revisión bibliográfica. Revista española de documentación científica, 13(3), 842-865.

Sacristán, J. A., y Dilla, T. (2019). El valor de las innovaciones médicas. Papeles de Economía Española, (160), 165-210. 
Seltzer, A. J., y Hamermesh, D. S. (2018). Co-authorship in economic history and economics: Are we any different?. Explorations in Economic History, 69, 102-109.

Sciendo Website Remav (s.f) Real Estate Management and Valuation. Sciendo Website Remav

Simoes, N., y Crespo, N. (2020). Self-Citations and scientific evaluation: Leadership, in- fluence, and performance. Journal of Informetrics, 14(1), 100990.

Whitehead, M. (2014). Price of everything/ value of nothing. Environmental Values, 23(3), 249-252.

Žukauskas, V., y Hülsmann, J. G. (2019). Financial asset valuations: The total demand approach. The Quarterly Review of Economics and Finance, 72, 123-131. 